\title{
Proceedings of the 10th Alcohol Hangover Research Group Meeting in Utrecht, The Netherlands
}

\author{
Agnese Merlo 1, Zack Abbott ${ }^{2}$, Chris Alford ${ }^{3}$, Stephanie Balikji ${ }^{4}$, Gillian Bruce 1, Craig Gunn 5 , \\ Jacqueline Iversen 6, Jim Iversen 6, Sean J. Johnson 3,7, L. Darren Kruisselbrink 8, \\ Aurora J. A. E. van de Loo ${ }^{4,9}$, Marlou Mackus ${ }^{4}$, Chantal Terpstra ${ }^{10}$, Ann-Kathrin Stock 11 \\ and Joris C. Verster $4,9,10, *$ \\ 1 Education and Social Sciences, University of the West of Scotland, Paisley PA1 2BE, UK; \\ agnese.merlo@gmail.com (A.M.); gillian.bruce@uws.ac.uk (G.B.) \\ 2 ZBiotics Company, 181 2nd Street, San Francisco, CA 94105, USA; zack@zbiotics.com \\ 3 Psychological Sciences Research Group, University of the West of England, Bristol BS16 1QY, UK; \\ chris.alford@uwe.ac.uk (C.A.); JohnsonS11@cardiff.ac.uk (S.J.J.) \\ 4 Division of Pharmacology, Utrecht Institute for Pharmaceutical Sciences (UIPS), Utrecht University, \\ 3584CG Utrecht, The Netherlands; stephaniebalikji@hotmail.com (S.B.); a.j.a.e.vandeloo@uu.nl \\ (A.J.A.E.v.d.L.); marloumackus@gmail.com (M.M.) \\ 5 Addiction and Mental Health Group, Department of Psychology, University of Bath, Bath BA2 7AY, UK; \\ cag35@bath.ac.uk \\ 6 Sen-Jam Pharmaceutical, 223 Wall Street \#130, Huntington, NY 11743, USA; jackie@sen-jam.com (J.I.); \\ jiversen@sen-jam.com (J.I.) \\ 7 Centre for Trials Research, Cardiff University, Cardiff CF14 4YS, UK \\ 8 Centre of Lifestyle Studies, School of Kinesiology, Acadia University, Wolfville, NS B4P 2R6, Canada; \\ darren.kruisselbrink@acadiau.ca \\ 9 Institute for Risk Assessment Sciences (IRAS), Utrecht University, 3584CM Utrecht, The Netherlands \\ 10 Centre for Human Psychopharmacology, Swinburne University, Melbourne, VIC 3122, Australia; \\ chantalterpstra92@gmail.com \\ 11 Cognitive Neurophysiology Department of Child and Adolescent Psychiatry, \\ Faculty of Medicine of the TU Dresden, University of Dresden, D-01307 Dresden, Germany; \\ Ann-Kathrin.Stock@uniklinikum-dresden.de \\ * Correspondence: j.c.verster@uu.nl; Tel.: +32-1302536909
}

Published: 28 March 2020

\begin{abstract}
The hangover is the most commonly reported negative consequence of alcohol use with several studies reporting the detrimental consequences of hangover on health, economy, and society. Research has emphasized the socioeconomic consequences of experiencing these physical and psychological symptoms in relation to absenteeism, increased risk of having accidents and injuries, and impairment of daily activities, such as job performance and driving a car. During the 10th Alcohol Hangover Research Group meeting, held on 29 April 2018, in Utrecht, The Netherlands, aspects of alcohol hangover were presented with regards to determinants, biological and cognitive consequences and potential treatments. Precursory and posterior factors influencing alcohol hangover, including biological, psychological, behavioral, metabolic aspects, cognitive functioning, and the role of the immune system in the development of alcohol hangover, were presented. In addition, potential preventive measures and treatments of alcohol hangover to reduce the adverse consequences of alcohol consumption and hangover symptoms were discussed. One study revealed that an average of $24 \%$ of social and heavy drinkers claimed not to experience hangover symptoms across time. Another study showed that food intake (either healthy or junk food) had no significant impact on next-day hangover severity. Research examining cognitive and psychomotor functioning during hangover revealed impairments in collective problem solving and response inhibition, but not attentional bias towards alcohol-related cues. The alcohol hangover
\end{abstract}


state further significantly impaired driving performance, even for a short commute to work. With regard to the pathology of the alcohol hangover, research was presented that demonstrated increases in saliva cytokine concentrations confirming drinking alcohol and the hangover phase are both associated with an immune response. Other presentations discussed that scientific literature shows that there are no effective hangover treatments available yet. However, although promising, new hangover treatments are currently in development. Taken together, at the 10th Alcohol Hangover Research Group meeting, a comprehensive overview of the causes, consequences, and potential treatments of the alcohol hangover was presented.

Keywords: alcohol; hangover; causes; consequences; treatment

\section{Introduction}

Alcohol hangover is the most commonly experienced consequence of alcohol consumption [1] and is defined as the combination of negative mental and physical symptoms which can be experienced after a single episode of alcohol consumption, starting when blood alcohol concentration (BAC) approaches zero [2,3]. Alcohol hangovers are associated with an increased risk of having accidents and injury and the impaired performance of daily activities, and the corresponding workrelated costs in terms of absenteeism and presenteeism to the economy and society are high [4]. To support alcohol hangover related research, in 2010 the Alcohol Hangover Research Group (AHRG) was established to promote international research and collaboration, review and enhance methodological procedures and increase general knowledge of alcohol hangover. Up to now several AHRG meetings have been held to discuss the causes, consequences and treatment of the alcohol hangover [5-9]. The present proceedings provide a synopsis of the 10th AHRG meeting, held on 29 April 2018, in Utrecht, The Netherlands.

\section{Precursory Factors of Alcohol Hangover}

Research suggests a correlation between certain personality traits and risk for alcoholism, as well as reduced or increased presence and the severity of hangover symptoms [10]. Although lower sensitivity to alcohol is only present in a minority of the population, this distinction has drawn researchers' attention, as resistance to hangover has been shown to be correlated with engagement of excessive drinking and to be a risk factor for alcohol use disorder [11].

Agnese Merlo (University of the West of Scotland, UK) discussed differences in mood and personality among hangover resistant and hangover sensitive social drinkers. Previous and current literature has observed and investigated two categories of drinkers, hangover sensitive and hangover resistant. Howland et al. [12] investigated hangover resistance among six survey studies which implemented different designs and populations. It was revealed that an average of $24 \%$ of social and heavy drinkers, claimed not to experience hangover symptoms across time [12]. In Merlo's study, social drinkers were invited to complete an online survey. Estimated BAC on their past month's heaviest drinking occasion was calculated to confirm social drinkers that achieved an estimated BAC of at least $0.11 \%$. The latter was done to ensure that participants consume enough alcohol to develop a hangover per se. $\mathrm{N}=986$ social drinkers met this criterion and were allocated to either the hangover sensitive group $(N=893)$ or the hangover resistant group $(N=93)$, based on whether they experienced a hangover during the past year. All participants completed the Short-Form Profile of Mood State (POMS-SF), the neuroticism scale of the Eysenck Personality Questionnaire (EPQ), and the Depression Anxiety Stress Scale (DASS-21). Hangover resistant drinkers scored significantly lower on DASS-21 stress and anxiety, POMS-SF depression, and the EPQ neuroticism scale. The two groups also differed significantly in alcohol consumption patterns (both frequency and quantity) and estimated BAC, which were significantly higher in hangover sensitive drinkers. Re-analyzing the data with estimated BAC as covariate revealed that compared to hangover sensitive drinkers, hangover resistant drinkers score significantly lower on the DASS-21 scales for stress and anxiety, 
and the EPQ neuroticism scale. In conclusion, significant mood and personality changes are observed between hangover sensitive and resistant drinkers. Further research is needed to elucidate the impact of mood and personality on having hangovers.

Gillian Bruce (University of the West of Scotland, UK) presented preliminary data investigating the motives for drinking in hangover sensitive and hangover resistant drinkers. It has been suggested that drinking motives may represent the most rudimentary psychological factors that lead to alcohol consumption decisions being made [13] and while a number of studies have shown associations between drinking motives and alcohol use [14], it would appear that drinking motives and alcohol hangover have not been examined. Bruce discussed initial findings of a study which investigated differences in drinking motives between individuals who were hangover sensitive and those who were hangover resistant. For reasons outlined above, participants were only included in analyses if their estimated BAC on their past month's heaviest drinking occasion reached $0.11 \%$ or higher. Preliminary analysis suggests drinking motives to be significantly higher in hangover sensitive participants. While these data add to the evidence that psychosocial differences exist between hangover sensitive and resistant drinkers, additional analysis and further research is needed to examine the factors underpinning to this difference.

\section{Behavioral Aspects of Alcohol Hangover}

Behavioral aspects covered during the meeting include innovative measures of alcohol hangovers, which involve the use of social media to gather real-time data and increase ecological validity of alcohol hangover research. Moreover, the role played by specific nutrients and types of food on the presence and effects of alcohol hangover was examined.

Sean Johnson (University of the West of England, UK) discussed contemporary behavioral measures of alcohol hangover. Previous research has typically investigated the alcohol hangover by asking alcohol consumers to report on their drinking practices and hangover severity following an evening of drinking. This approach is limited in that it usually involves next day retrospective recall of drinking during one occasion, which may be compromised by inaccuracy in reporting due to memory loss [15]. Sean Johnson presented exploratory findings from a novel methodology of utilizing participants social media applications to collect real-time alcohol consumption data over a six-week period. Participants also reported risk-taking behavior, subjective intoxication and next day hangover severity. The study was conducted amongst 101 university students and demonstrated feasibility in collecting longitudinal alcohol consumption data with $80.2 \%$ of participants providing at least two drinking occasions during the six-week period. Of all possible days, $20.8 \%$ included the consumption of alcohol, with participants reporting an average of eight drinking occasions across the six-week period. An alcohol hangover was reported on $74.2 \%$ of all drinking occasions. Alcohol hangover occasions were typically characterized by greater alcohol consumption (greater number of alcohol units consumed, higher estimated BAC and subjective intoxication), the consumption of spirits, a longer duration of drinking occasion, and attendance at late night entertainment venues. Prospective methodologies present the opportunity to capture in-depth alcohol hangover data, however this must be balanced with the cost and time needed to conduct such studies.

Stephanie Balikji (Utrecht University, The Netherlands), aimed at examining the impact of consuming healthy food, junk food, or no food, either before, during or after an evening of heavy alcohol consumption [16]. $\mathrm{N}=435$ Dutch students were invited on Facebook to complete an online survey. Alcohol consumption data was collected for their latest past month heavy drinking session, including the number of alcoholic and non-alcoholic drinks consumed, and the duration of the drinking session. Subjective intoxication and next-day hangover severity were both rated. Participants could indicate whether they consumed healthy food, junk food, or no food, before, during or after the heavy drinking session. The conclusion was that junk food and healthy food have a differential time-dependent effect on the number of alcoholic and non-alcoholic beverages consumed, and levels of subjective intoxication, but food intake (either healthy or junk food) had no significant impact on next-day hangover severity. 


\section{Cognitive Effects of Alcohol Hangover}

Alcohol hangover is associated with impaired psychomotor skills in relation to driving [17-19] and cognitive impairment has been observed in relation to alcohol hangover, such as impaired memory retrieval, negative mood, and attention deficits [20-26]. The following studies review cognitive and motor impairment during alcohol hangover.

Darren Kruisselbrink (Acadia University, Canada) reviewed the hangover literature to determine patterns of neurocognitive impairment and non-impairment in hangover research. Authors of existing reviews of hangover research have grouped research findings in various ways to describe clusters of findings [20-26] and have provided the most structured review, classifying the research they reviewed into the broad areas of attention and memory, each with more narrowly defined sub-areas. Kruisselbrink used the neurocognitive domains and subdomains outlined in the Diagnostic and Statistical Manual of Mental Disorders - 5th edition (DSM-5) to structure his review since this classification system is widely recognized in the fields of psychology and psychiatry [27]. It will allow hangover researchers to see which neurocognitive domains and subdomains have been subjected to more and less research, and how these domains and subdomains are affected by hangover. The six domains listed by the DSM- 5 include complex attention, executive function, learning and memory, perceptual-motor function, language, and social cognition; each domain includes a number of subdomains. In each of the 35 studies Kruisselbrink reviewed, he assessed the neurocognitive demands for each experimental task, assigned it to a DSM-5 subdomain (and, by definition, domain), and categorized performance during hangover as either impaired or not impaired. The pattern of results showed that more hangover research has been conducted in the neurocognitive domains of attention, executive function, perceptual-motor function, and learning and memory than in the domains of language and social cognition. Furthermore, within each domain, some subdomains have received more research attention than others (See Figure 1).

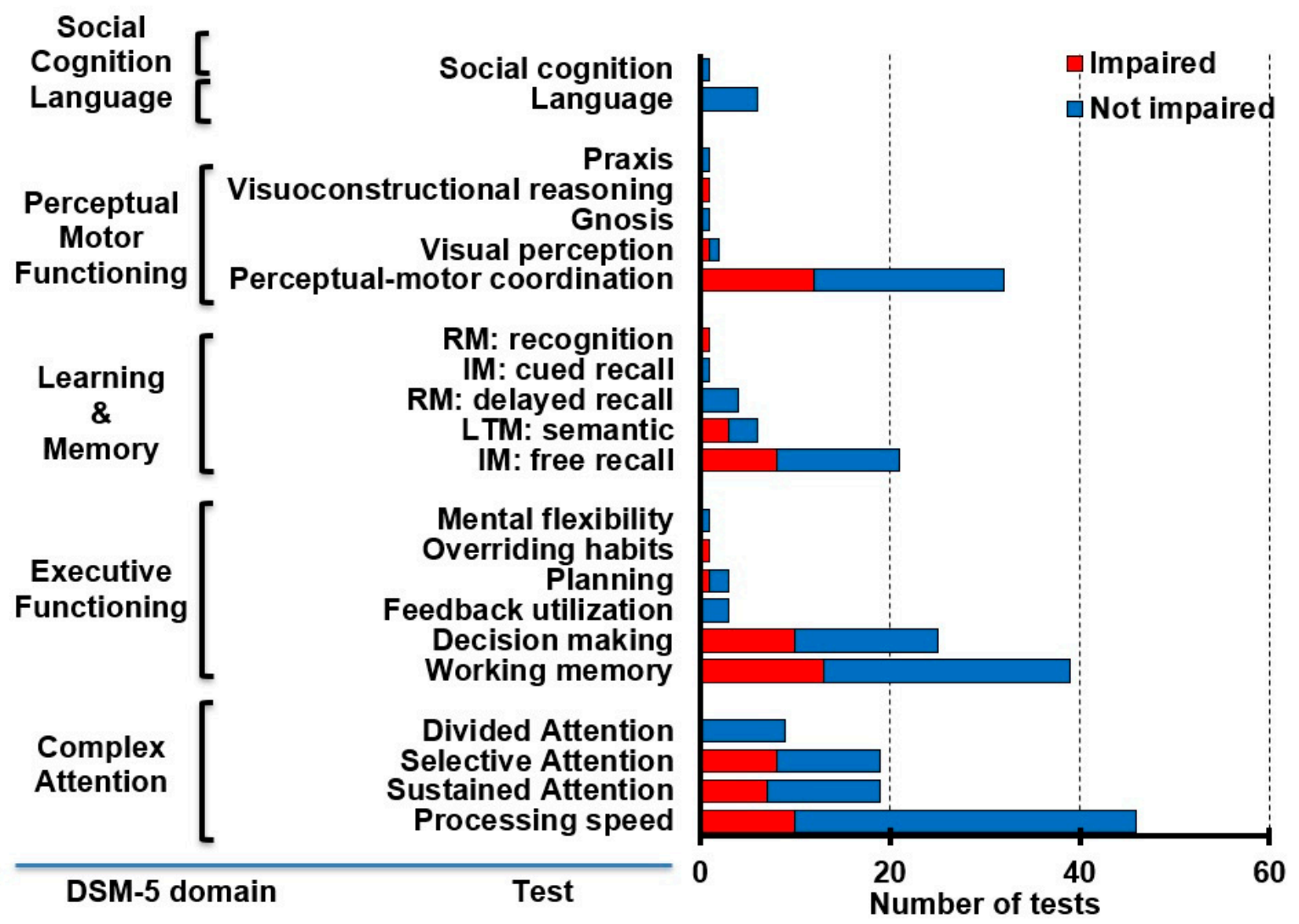

Figure 1. Frequency of impairment during hangover in DSM-5 neurocognitive subdomains. Abbreviations: DSM = Diagnostic and Statistical Manual of Mental Disorders, RM = recall memory, $\mathrm{IM}=$ immediate memory, $\mathrm{LTM}=$ long term memory. 
The patterns of impairment and non-impairment also showed that neurocognitive impairment as a function of hangover only occurred $22-42 \%$ of the time in subdomains that had been examined 10 or more times. The organization of hangover research results using the DSM- 5 framework demonstrates that (1) neurocognitive impairment due to the residual effects of alcohol appear somewhat challenging to find, suggesting a need to expand measures of impairment beyond speed and accuracy to include effort and fatigue; (2) almost all of the experimental tasks used by hangover researchers require individual rather than collective problem solving. Since many occupational environments require individuals to solve problems collectively, research examining collective problem solving may represent an important research opportunity for hangover researchers; and (3) currently some neurocognitive domains and subdomains are under-researched-notably the domain of social cognition-suggesting opportunities for hangover researchers to expand the hangover research database.

Chris Alford (University of the West of England, UK) discussed the impact of alcohol hangover on simulated driving during a 'commute to work', including a comparison between residual alcohol and residual no alcohol groups. Previous research showed that highway driving is significantly impaired during the hangover state $[17,18]$, and that despite this knowledge drivers continue driving while hungover [19]. Whilst an earlier study has assessed the impact on an hour-long driving simulation [18], the potential for impact on a shorter typical commute to work was investigated in the current study. A total of $\mathrm{N}=52$ participants (age range 18-25 years, mixed sex group) took part in a 20 minute (STISIM) simulated drive with a $50 \mathrm{mph}$ speed limit and including mixed urban and rural environments, as well as hazards such as pedestrians stepping into the road. They were assessed next morning following a night of social drinking (hangover condition) and after a night without alcohol (control condition). Participants were divided into two groups based on breathalyzer assessment. There were $\mathrm{N}=26$ participants who had zero alcohol $(0 \% \mathrm{BAC})$ and $\mathrm{N}=26$ participants in the residual alcohol group who averaged just less than $0.05 \%$ BAC. Participants showed a significant level of hangover symptoms with the alcohol hangover severity scale and marked increases in four of the six NASA TLX perceived workload dimensions. Both zero and residual alcohol groups had slower response times to the divided attention task, though significantly greater driving speed. Relatively increased center line crossing and time off road, reflecting poorer control, were also seen for the residual alcohol group. Taken together, these results show that the alcohol hangover state significantly impairs driving performance, even for a relatively short 'commute to work' and despite a greater subjective effort being made by drivers. The findings have implications for policy and public awareness - indicating significantly impaired driving despite being 'street legal' with a breathalyzer.

Craig Gunn (University of Bath, UK) presented preliminary data on an experimental study examining the effects of alcohol hangover on response inhibition and attentional bias towards alcohol-related stimuli [28]. Previous research has highlighted that few studies have explored how alcohol hangover influences executive functions and future alcohol use behaviors. Gunn et al, conducted a naturalistic study (participants engaged in a night of usual alcohol consumption, determining the quantity, type of beverage, and time of drinking) with a within-subjects, counterbalanced design. Participants were breathalyzed to ensure that BAC was below $0.02 \%$ before testing began and completed pre- and post-cognitive task measures of mood, hangover, sleep, alcohol craving, and perceived mental effort. Participants also completed two computerized cognitive tasks exploring response inhibition $(\mathrm{Go} / \mathrm{No}-\mathrm{Go})$ and attentional bias towards alcohol-related stimuli (Visual Dot Probe). Preliminary results ( $\mathrm{n}=33$ to date) were presented for cognitive data on response inhibition and attentional bias towards alcohol-related stimuli. In the Go/No-Go task, participants made more commission errors (failures to inhibit) in the hangover condition compared to the nohangover condition. In the visual dot probe task, participants had slower reactions times in the hangover condition compared to the no-hangover condition, but this was not influenced by cue type (i.e., alcohol versus neutral). Together, these data suggest that hangover impairs response inhibition, but not attentional bias towards alcohol-related cues. Future research should explore other components of executive function (e.g., shifting, updating) during hangover, as inhibition may be a 
'common factor' underlying other cognitive processes. In addition, behaviors that are important for everyday activities, which rely on executive functions (e.g., emotion regulation), should be explored during hangover.

Chantal Terpstra (Swinburne University, Australia), discussed the effects of a hangover on attentional resources. Based on previous research $[25,26]$, it was hypothesized that a hangover would impair performance measured during dual attention tasks. Hangovers were expected to result in higher effort ratings following cognitive testing and to impair more effortful cognitive tasks. Furthermore, hangover was predicted to reduce neural efficiency and therefore increase ratings of effort during task performance. A total of 23 drinkers (study not completed yet) participated in a naturalistic study consisting of a hangover day (alcohol consumed) and a control day (no alcohol consumed). Data were collected on alcohol consumption, demographics, sleep, next-day adverse effects, mood and perceived effort (physical and mental), frustration and performance. Participants completed a dual attention task (on control day and hangover day). This consisted of a verbal memory task with psychomotor tracking during one half of the auditory presentation phase. Accuracy and reaction times were recorded during a visual word recognition phase. There were main effects of both tracking and hangover. There was also a main effect of hangover, but not tracking, on response time. There was no tracking $x$ hangover interaction for either measure. Additionally, National Aeronautics and Space Administration - Task Load Index (NASA-TLX) scores indicated greater subjective difficulty and need for more resources. This profound level of impairment has clear implications for day-to-day activities and behaviors.

Ann-Kathrin Stock (TU Dresden, Germany) presented the preliminary results of a hangover study investigating the effects of an experimentally induced hangover state on cognitive control in healthy young male subjects [29]. Specifically, the study investigated the effect of conflict load (which was induced with the help of a masked subliminal prime) on cognitive control (which was induced with the help of a flanker stimuli) in a counter-balanced within-subject design. As a previous study had demonstrated that the speed of information accumulation from visual stimuli seems to be accelerated when hungover [30], it was hypothesized that subliminal conflicts might increase and therefore cause a stronger behavioral interference when hungover. Even though cognitive control conflicts and conflict adaptation were not generally impaired during a hangover state, the results demonstrated that conflict load (i.e., an underlying conflict induced via subliminal primes) attenuated cognitive control functions (as reflected by the size of the flanker effect) more during a hangover than during a sober state. This finding matches the assumption of faster information accumulation during hangover, as a faster/deeper processing of subliminal primes should lead to greater conflicts and therefore hamper cognitive control via an increased conflict load. It was further speculated whether this effect could be linked to the GABAergic changes associated with alcohol hangover [29-36]. Interestingly, the size of the observed effect was correlated with age and subjective sleepiness, but neither with peak breath alcohol concentrations during the experimentally induced intoxication, nor with subjective ratings of general hangover symptoms. The lack of effects in those two dimensions could however have been caused by the standardized experimental alcohol administration procedure that had been designed to minimize the variance in all intoxication-related parameters.

\section{Biological Effects of Alcohol Hangover}

In addition to the psychological, cognitive, and behavioral factors that influence the severity of hangovers, biological underpinnings of hangovers are crucial to the understanding of this physical and psychological induce state. Research to reveal these biological mechanisms, which was presented during the AHRG meeting, is summarized here.

Aurora van de Loo (Utrecht University, The Netherlands), discussed the change in cytokine concentration, both acutely and during the hangover. In previous studies, changes in cytokine concentrations both after drinking alcohol [37] and during the alcohol hangover phase [38] have been reported in blood, suggesting a role for the immune system. The preliminary results of two studies were presented, both regarding the cytokine changes in saliva. The first study focused on measuring cytokine concentrations directly after consuming alcohol and after the consumption of a placebo, 
every hour for eight hours. The second study aimed at measuring salivary cytokine responses the morning after an evening of drinking alcohol, i.e. during the hangover phase, and the morning on a control day. Cytokine concentrations that could be reliably assessed (Interleukin (IL)-1 $\beta$, IL-8, Tumor Necrosis Factor (TNF)- $\alpha$ in both studies, and IL-6, IL-10 in the hangover study only) were compared between the test day and the control day. Directly after consuming alcohol significant increases in cytokines IL-1 $\beta$ and Il- 8 were found on multiple time points during the day. During the hangover phase cytokine concentrations of IL-6, IL-10, and TNF- $\alpha$ were significantly increased. After adjusting for multiple comparisons, the increases in concentration were significant for IL- 6 and IL-10. These increases in saliva cytokine concentrations confirm that drinking alcohol and the hangover phase are both associated with the immune system. The exact role of the immune system is still unclear.

Marlou Mackus (Utrecht University, The Netherlands), discussed the role of metabolites as indicators of alcohol hangover. The alcohol hangover being the most commonly experienced sideeffect of excessive alcohol consumption and the demand for an effective hangover cure requires the identification of sensitive and reliable biomarkers. Previous research has shown a shift in the ratio of two serotonin metabolites, 5-hydroxytryptophol (5-HTOL) and 5-hydroxyindol-3-ylacetic acid (5-HIAA), after recent alcohol consumption. As both metabolites are still present in urine even after ethanol has been cleared from the body, these are thought to be potential indicators of the alcohol hangover. In the presented study [39], on a hangover day and alcohol-free control day, urine samples were collected in the morning. Urine samples of $\mathrm{N}=36$ participants ( 18 hangover and 18 hangoverresistant) were analyzed on the concentration of 5-HTOL and 5-HIAA. Urine samples were analyzed by performing a gradient high-performance liquid chromatography (HPLC) Fluorescent detection method, using an acetonitrile buffer $(\mathrm{pH}=5.5)$ and hydroxyindole-3-propionic acid (HIPA) as internal standard. Each sample was treated with beta-glucuronidase and extracted with diethyl ether to optimize yield. The urinary concentration of both 5-HIAA and 5-HTOL were significantly higher on the post-alcohol day, compared to control levels $(p<0.001)$. The 5-HTOL:5-HIAA ratio was also found to be significantly higher in the post-alcohol condition. In the whole population, the urinary concentrations of 5-HTOL on the post-alcohol day did not significantly correlate with the total hangover score or the 1-item hangover score, nor did the ratio of 5-HTOL:5-HIAA. Only the urinary concentration of 5-HIAA on post-alcohol day was shown to significantly correlate with the total hangover score. When analyzing the data for the subset of hangover sensitive drinkers only, none of the correlations were statistically significant. The results of this study confirm that the ratio of 5-HTOL:5-HIAA is a reliable biomarker of recent heavy alcohol consumption. However, the 5-HTOL:5-HIAA ratio is not associated with the severity of the alcohol hangover, or its individual symptoms.

\section{Interventions and Treatments for Alcohol Hangover}

Joris Verster (Utrecht University, The Netherlands) discussed research conducted among a large sample of Dutch students who regularly experience alcohol hangovers [40]. This study investigated the desirability for an effective hangover treatment. The study showed that a majority of drinkers desire the availability of an effective hangover treatment and attest that this will not alter their drinking behavior. The most important reason is that drinkers want to both enjoy the drinking occasion and perform optimally the next-morning. Current scientific literature shows that there are no effective hangover treatments available yet [41-43]. Only a fraction of the marketed hangover products have been scientifically investigated, and for none of these products is substantial scientific evidence (i.e., randomized controlled trials in human subjects) available that demonstrates their efficacy in treating or preventing alcohol hangover.

Jim Iversen (Sen-Jam Pharmaceutical, USA) discussed the difficulties of obtaining research funding to examine the efficacy and safety of hangover products. The traditional pharmaceutical companies have no hangover products in development, and companies that do develop these products are usually start-up companies with limited financial resources. Of concern, as most of these companies plan to market their hangover product as dietary supplements, no research is required by the US Food and Drug Administration (FDA) to demonstrate safety and efficacy. Given the great 
socioeconomic costs of alcohol hangover, it is important that investments are made to develop and test the efficacy of new hangover treatments.

Jacqueline Iversen (Sen-Jam Pharmaceutical, USA) discussed the development of a new treatment for alcohol hangover. Alcohol consumption causes the release of pro-inflammatory agents, which are implicated in causing the associated alcohol derived hangover the next day. Based on this research, Sen-Jam Pharmaceutical, has created a fixed dose combination product, incorporating potent, yet safe, anti-inflammatory agents together (i.e., a non-steroidal anti-inflammatory and an antihistamine drug), in an oral dosage form for the prevention of alcohol hangover when administered prior to consuming alcohol. A US patent has been awarded in September 2019, and clinical trials are underway.

Zack Abbott (ZBiotics Company, USA) discussed reducing exposure to acetaldehyde with engineered probiotics that robustly express a bacterial acetaldehyde dehydrogenase (ALDH). Acetaldehyde is a known carcinogen that causes DNA damage, cell death, and inflammation [44,45]. It has been demonstrated to accumulate as a result of ethanol ingestion. Commonly it is thought to result from incomplete metabolism of alcohol in the liver, but the highest concentration of acetaldehyde is found in the colon following alcohol ingestion [46]. This is believed to be the result of microbial and gut epithelial alcohol dehydrogenases, which are uncoupled from aldehyde dehydrogenases [46]. It is hypothesized that this may be a major source of systemic acetaldehyde. To counteract this, we have engineered probiotic bactera to constitutively and robustly express a bacterial acetaldehyde dehydrogenase. The purpose of this would be for a person to ingest the engineered probiotic during an event of alcohol ingestion so that the bacteria could help to rapidly oxidize acetaldehyde into acetate in the gut as it accumulates, reducing systemic levels and limiting the damage and some of the symptoms associated with a hangover. Abbott et al. demonstrated in vitro that the engineered bacteria can indeed reduce acetaldehyde at physiologically relevant rates. ZBiotics is in the process of assembling a generally recognized as safe (GRAS) dossier to be filed with the US FDA for the purpose of bringing the engineered probiotic to market as a food product. Further, a mouse study will be conducted to quantify the effects of the probiotic on acetaldehyde levels in vivo, and a human study to assess the probiotic's efficacy in preventing hangovers.

\section{Discussion}

Various aspects of alcohol hangover were discussed during the 10th AHRG meeting. Specifically advances in relation to potential determinants, cognitive and motor consequences, as well as intervention and treatment of alcohol hangover were presented alongside data that advanced knowledge in biological and psychosocial domains of alcohol hangover. Further evidence, for example, of cognitive impairment during the alcohol hangover was discussed together with findings on personality and mood, suggesting that we are slowly building a more coherent picture on the psychological effects of the alcohol hangover. Data were also presented showing differences in motives for drinking in hangover resistant and hangover sensitive drinkers, suggesting that psychological factors may be influential in predicting hangover resistance. Furthermore, a number of speakers provided new data on the biological implications of alcohol hangover including an increase in cytokine levels and the reliability of the presence of certain biomarkers. The final strand of presentations focused on the advances in developing products to cure/prevent the alcohol hangover.

An important issue raised was the lack of funding of alcohol hangover research despite the significant and copious evidence to support the negative consequences of alcohol hangover, which affect not only our economy, but also our society and well-being. Alcohol induced hangovers are the most common reported consequence of alcohol consumption at a global level, thus identifying its underlying biological and psychological mechanism, as well as generate an effective preventative measure or treatment would aid in reducing related costs and adverse social consequences.

Taken together, at the 10th Alcohol Hangover Research Group Meeting a variety of important new research topics have been discussed. Over the past year, significant progress has been made to enhance the research field. New international collaborations were set-up to further strengthen research in the field of alcohol hangover. 
Author Contributions: Conceptualization and design, A.M. and J.C.V.; writing original draft: A.M., G.B. and J.C.V.; all authors critically reviewed the manuscript for important intellectual content and approved the final version. All authors have read and agreed to the published version of the manuscript.

Funding: This 10th Alcohol Hangover Research Group meeting was financially supported by Utrecht University.

Conflicts of Interest: C.A. has undertaken sponsored research, or provided consultancy, for a number of companies and organizations including Airbus Group Industries, Astra, British Aerospace/Bae Systems, Civil Aviation Authority, Duphar, Farm Italia Carlo Erba, Ford Motor Company, ICI, Innovate UK, Janssen, LERS Synthélabo, Lilly, Lorex/Searle, UK Ministry of Defense, Quest International, Red Bull GmbH, Rhone-Poulenc Rorer, and Sanofi Aventis. S.J. has undertaken sponsored research for Pfizer, AstraZeneca, Merck, Gilead, Novartis, Roche, Red Bull GmbH, the Department for Transport, and Road Safety Trust. A.K.S. has received a Daimler and Benz Foundation scholarship for the promotion of postdoctoral students and assistant professors. Over the past 3 years, J.C.V. has received grants/research support from the Dutch Ministry of Infrastructure and the Environment, Janssen Research and Development, and Sequential, and has acted as a consultant/advisor for Clinilabs, More Labs, Red Bull, Sen-Jam Pharmaceutical, Toast!, and ZBiotics. Z.A. is founder and CEO of ZBiotics. J.I. (Jacqueline Iversen) and J.I. (Jim Iversen) are founders of Sen-Jam Pharmaceutical. The other authors have no potential conflicts of interest to disclose.

\section{References}

1. Verster, J.C.; van Herwijnen, J.; Olivier, B.; Kahler, C.W. Validation of the Dutch version of the brief young adult alcohol consequences questionnaire (B-YAACQ). Addict. Behav. 2009, 34, 411-414.

2. Van Schrojenstein Lantman, M.; van de Loo, A.J.A.E.; Mackus, M.; Verster, J.C. Development of a definition for the alcohol hangover: Consumer descriptions and expert consensus. Curr. Drug Abuse Rev. 2016, 9, 148-154.

3. Verster, J.C.; Scholey, A.; van de Loo, A.J.A.E.; Benson, S.; Stock, A.K. Updating the definition of the alcohol hangover. J. Clin. Med. 2020, 9, 823.

4. Bhattacharya, A. Financial Headache. The Cost of Workplace Hangovers and Intoxication to the UK Economy; Institute of Alcohol Studies: London, UK, 2019.

5. Merlo, A.; Adams, S.; Benson, S.; Devenney, L.; Gunn, C.; Iversen, J.; Johnson, S.J.; Mackus, M.; Scholey, A.; Stock, A.K.; et al. Proceedings of the 9th Alcohol Hangover Research Group Meeting. Curr. Drug Abuse Rev. 2017, 10, 68-75.

6. Verster, J.C.; Stephens, R. The importance of raising the profile of alcohol hangover research. Curr. Drug Abuse Rev. 2010, 3, 64-67.

7. Howland, J.; Rohsenow, D.J.; McGeary, J.E.; Streeter, C.; Verster, J.C. Proceedings of the 2010 symposium on hangover and other residual alcohol effects: Predictors and consequences. Open Addict. J. 2010, 3, 131-132.

8. Verster, J.C.; Alford, C.; Bervoets, A.C.; de Klerk, S.; Grange, J.; Hogewoning, A.; Jones, K.; Kruisselbrink, D.; Owen, L.; Piasecki, T.M.; et al. The Alcohol Hangover Research Group. Hangover research needs: Proceedings of the 5th Alcohol Hangover Research Group meeting. Curr. Drug Abuse Rev. 2013, 6, $245-251$.

9. Mackus, M.; Adams, S.; Barzilay, A.; Benson, S.; Blau, L.; Iversen, J.; Johnson, S.J.; Keshavarzian, A.; Scholey, A.; Smith, G.S.; et al. Proceeding of the 8th Alcohol Hangover Research Group meeting. Curr. Drug Abuse Rev. 2016, 9, 106-112.

10. Swift, R.; Davidson, D. Alcohol hangover. Alcohol Health Res. World 1998, 22, 54-60.

11. Piasecki, T.M.; Alley, K.J. Low sensitivity to alcohol: Relations with hangover occurrence and susceptibility in an ecological momentary assessment investigation. J. Stud. Alcohol Drugs 2012, 73, 925-932.

12. Howland, J.; Rohsenow, D.J.; Edwards, E.M. Are some drinkers resistant to hangover? A literature review. Curr. Drug Abuse Rev. 2008, 1, 42-46.

13. Cox, W.M.; Klinger, E. A motivational model of alcohol use. J. Abnorm. Psychol. 1988, 97, 168-180.

14. Bruce, G.; Curren, C.; Williams, L. Alexithymia and alcohol consumption: The mediating effects of drinking motives. Addict. Behav. 2012, 37, 350-352.

15. Verster, J.C.; van de Loo, A.J.A.E.; Adams, S.; Stock, A.K.; Benson, S.; Alford, C.; Scholey, A.; Bruce, G. Advantages and limitations of naturalistic study designs and their implementation in alcohol hangover research. J. Clin. Med. 2019, 8, 2160. 
16. Verster, J.C.; Stam, N.; Sterk, A.Y.; van de Loo, A.J.A.E.; Mackus, M.; Balikji, S.; Kraneveld, A.D.; Scholey, A.; Garssen, J. Food intake before, during, and after a heavy drinking session. Alcohol. Clin. Exp. Res. 2018, 42 (Suppl. 1), 52A.

17. Verster, J.C. Alcohol hangover effects on driving and flying. Int. J. Disabil. Hum. Dev. 2007, 6, 361-367.

18. Verster, J.C.; Bervoets, A.C.; de Klerk, S.; Vreman, R.A.; Olivier, B.; Roth, T.; Brookhuis, K.A. Effects of alcohol hangover on simulated highway driving performance. Psychopharmacology 2014, 231, 2999-3008.

19. Verster, J.C.; van Der Maarel, M.A.; McKinney, A.; Olivier, B.; de Haan, L. Driving during alcohol hangover among Dutch professional truck drivers. Traffic. Inj. Prev. 2014, 15, 434-438.

20. Prat, G.; Adan, A.; Pérez-Pàmies, M.; Sànchez-Turet, M. Neurocognitive effects of alcohol hangover. Addict. Behav. 2008, 33, 15-23.

21. Mc Kinney, A.; Coyle, K. Alcohol hangover effects on measures of affect the morning after a normal night's drinking. Alcohol Alcohol. 2006, 41, 54-60.

22. Barker, C.T. The alcohol hangover and its potential impact on the UK armed forces: A review of the literature on post-alcohol impairment. J. R. Army Med. Corps 2004, 150, 168-174.

23. Lemon, J. Alcoholic hangover and performance: A review. Drug Alcohol Rev. 1993, 12, $299-314$.

24. Ling, J.; Stephens, R.; Heffernan, T.M. Cognitive and psychomotor performance during alcohol hangover. Curr. Drug Abuse Rev. 2010, 3, 80-87.

25. Stephens, R.; Ling, J.; Heffernan, T.M.; Heather, N.; Jones, K. A review of the literature on the cognitive effects of alcohol hangover. Alcohol Alcohol. 2008, 43, 163-170.

26. Stephens, R.; Grange, J.A.; Jones, K.; Owen, L. A critical analysis of alcohol hangover research methodology for surveys or studies of effects on cognition. Psychopharmacology 2014, 231, 2223-2236.

27. Psychiatric Association. Diagnostic and Statistical Manual of Mental Disorders: Diagnostic and Statistical Manual of Mental Disorders, 5th ed.; American Psychiatric Association: Arlington, VA, USA, 2013.

28. Gunn, C.; Fairchild, G.; Verster, J.C.; Adams, S. The effects of alcohol hangover on response inhibition and attentional bias towards alcohol-related stimuli. Alcohol. Clin. Exp. Res. 2019, 43 (Suppl. 1), 185A.

29. Zink, N.; Bensmann, W.; Beste, C.; Stock, A.K. Alcohol hangover increases conflict load via faster processing of subliminal information. Front. Hum. Neurosci. 2018, 12, 316.

30. Stock, A.K.; Hoffmann, S.; Beste, C. Effects of binge drinking and hangover on response selection sub processes-A study using EEG and drift diffusion modeling. Addict. Biol. 2017, 22, 1355-1365.

31. Correa, M.; Salamone, J.D.; Segovia, K.N.; Pardo, M.; Longoni, R.; Spina, L.; Peana, A.T.; Vinci, S.; Acquas, E. Piecing together the puzzle of acetaldehyde as a neuroactive agent. Neurosci. Biobehav. Rev. 2012, 36, 404-430.

32. Enrico, P.; Sirca, D.; Mereu, M.; Peana, A.T.; Lintas, A.; Golosio, A.; Diana, M. Acetaldehyde sequestering prevents ethanol-induced stimulation of mesolimbic dopamine transmission. Drug Alcohol Depend. 2009, 100, 265-271.

33. Foddai, M.; Dosia, G.; Spiga, S.; Diana, M. Acetaldehyde increases dopaminergic neuronal activity in the VTA. Neuropsychopharmacology 2004, 29, 530-536.

34. Martí-Prats, L.; Sánchez-Catalán, M.J.; Orrico, A.; Zornoza, T.; Polache, A.; Granero, L. Opposite motor responses elicited by ethanol in the posterior VTA: The role of acetaldehyde and the non-metabolized fraction of ethanol. Neuropharmacology 2013, 72, 204-214.

35. Melis, M.; Enrico, P.; Peana, A.T.; Diana, M. Acetaldehyde mediates alcohol activation of the mesolimbic dopamine system. Eur. J. Neurosci. 2007, 26, 2824-2833.

36. Neupane, S.P. Neuroimmune interface in the comorbidity between alcohol use disorder and major depression. Front. Immunol. 2016, 7, 655.

37. Afshar, M.; Richards, S.; Mann, D.; Cross, A.; Smith, G.B.; Netzer, G.; Kovacs, E.; Hasday, J. Acute immunomodulatory effects of binge alcohol ingestion. Alcohol 2015, 49, 57-64.

38. Kim, D.J.; Kim, W.; Yoon, S.J.; Choi, B.M.; Kim, J.S.; Go, H.J.; Kim, Y.K.; Jeong, J. Effects of alcohol hangover on cytokine production in healthy subjects. Alcohol 2003, 31, 167-170.

39. Mackus, M.; van den Bogaard, W.J.M.; Korte-Bouws, G.A.H.; van de Loo, A.J.A.E.; Kraneveld, A.D.; Garssen, J.; Verster, J.C. 5HTOL/5HIAA ratio as biomarker of the alcohol hangover. Alcohol. Clin. Exp. Res. 2018, 42 (Suppl. 1), 37A.

40. Mackus, M.; van Schrojenstein Lantman, M.; van de Loo, A.J.A.E.; Nutt, D.J.; Verster, J.C. An effective hangover treatment: Friend or foe? Drug Sci. Policy Law 2017, doi:10.1177/2050324517741038. 
41. Pittler, M.H.; Verster, J.C.; Ernst, E. Interventions for preventing or treating alcohol hangover: Systematic review of randomised controlled trials. BMJ 2005, 331, 1515-1518.

42. Verster, J.C.; Penning, R. Treatment and prevention of alcohol hangover. Curr. Drug Abuse Rev. 2010, 3, 103-109.

43. Jayawardena, R.; Thejani, T.; Ranasinghe, P.; Fernando, D.; Verster, J.C. Interventions for treatment and/or prevention of alcohol hangover: A systematic review. Hum. Psychopharmacol. 2017, 32. doi:10.1002/ hup.2600.

44. Lambert, B.O.; He, S.M. DNA and chromosome damage induced by acetaldehyde in human lymphocytes in vitro. Ann. N. Y. Acad. Sci. 1988, 534, 369-376.

45. Šarc, L.; Lipnik-Štangelj, M. Comparison of ethanol and acetaldehyde toxicity in rat astrocytes in primary culture. Arh. Hig. Rada. Toksikol. 2009, 60, 297-305.

46. Salaspuro, M. Bacteriocolonic pathway for ethanol oxidation: Characteristics and implications. Ann. Med. 1996, 28, 195-200.

(C) 2020 by the authors. Licensee MDPI, Basel, Switzerland. This article is an open access article distributed under the terms and conditions of the Creative Commons Attribution (CC BY) license (http://creativecommons.org/licenses/by/4.0/). 